\title{
Sweet dreams: review of a sleep tracking framework
}

\begin{abstract}
Sleep disorders and deficiency are epidemic among young children and older adults. A 2016 US Centers for Disease Control and Prevention study revealed that more than a third of American adults and one out of four young American children lack sleep on a regular basis. To aid medical professionals investigating the etiology of sleep disorders, a number of environmental and physiological measurements are needed to understand this complicated state. With the development of wearable sensors, it is possible to monitor and track the effect of the environment outside the sleep clinics on an individual basis. A new sleep monitoring and tracking framework is presented which uses mobile platforms, computation resources, and sensors to create a home/ school/workplace-based monitoring and tracking environment. We conclude that this approach is useful for both adults and children, but that further work is needed to address current wearable sensor designs and performance as well as data quality, management, and security.
\end{abstract}

Keywords: sleep disorders, wearable sensors, internet of things, data security, mobile platform, real-time feedback, cloud, database usage
Volume 2 Issue 2 - 2017

\author{
Janet M Roveda, Kemeng Chen, Linda S \\ Powers \\ University of Arizona, USA
}

\begin{abstract}
Correspondence: Linda S Powers, Electrical and Computer Engineering, University of Arizona, 1230 E Speedway, Tucson, AZ, USA, Te: 520-621-7634, Fax 520-621-8076, Email Ispowers@email.arizona.edu
\end{abstract}

Received: March 01, 2017| Published: March 22, 2017
Abbreviations: CDC, centers for disease control internet of things; PSG, polysomnography; EOG, electrooculogram

\section{Introduction}

Sleep disorders result in sleep deficiency and are becoming epidemic issues among both young children and adults. A 2016 CDC study by the U.S. Centers for Disease Control and Prevention ${ }^{1}$ revealed that more than a third of American adults and one out of four young American children lack sufficient sleep on a regular basis. Another study conducted by the National Institute of Neurological Disorders and Stroke ${ }^{2}$ pointed out that every year, the US alone has over 40 million people suffering from chronic sleep disorders. Lack of sleep ${ }^{3}$ can cause detrimental health effects, such as increased probability for diabetes, neurological disorders, and heart disease as well as a weakened immune system. There is little doubt that sleep is essential for health, growth, normal physiological functions, and learning abilities in children.

The causes of sleep disorders are not well understood and are currently an active area of research. However, a myriad of environmental and physiological measurements are needed to understand this complicated physiological function. Unfortunately, sleep clinics can only provide snapshots of sleep tracking and monitoring, and fail to provide a continuous picture of sleep disorder related physiological measurements. Thus, it is imperative to find an effective way to monitor numerous physiological functions and sleep outside the clinical environment.

The proliferation of wearable sensors, Internet-of-Things (IoT), and the new technologies in system integration and designs have enabled us to make connections with physicians, caregivers, educators, parents, friends and a wide variety of real-time services without going to the hospital or clinic. It is possible to conduct tracking, monitoring and data streaming at the locations or in places we desire. This is especially important for sleep studies as we need to acquire sleep related measurements in places where users are in their normal living situations. $^{4-6}$
We point out that the 'in-place' concept emphasizes using

1) Reconfigurable designs and system integration ideas to establish an environment or mobile hub for persons-in-need at his/her own location, and

2) Stream the data to professionals at remote locations in real time through IoT.

This paper reviews sleep monitoring and tracking frameworks that can achieve 'in-place' data acquisition. We use mobile platforms, computational resources, and sensors to create a home/school/ workplace based tracking and monitoring environment. We also point out the potential challenges associated with the wearable sensor designs, data/measurement quality, and data management and security.

\section{Discussion}

We envision that sleep monitoring and tracking will follow two step transitions to aid accessible restful sleep for regular users at home/natural environment.

\section{Step I: From polysomnography (PSG) to wearables (e.g. wristband)}

PSG, performed at sleep centers or clinics, provides the most accurate medical diagnosis. However, due to its complexity in wiring and connections, clinic-based PSG test can only give snapshots of sleep data. Without the same level of accuracy, it is possible to use existing consumer based wearables to provide meaningful sleep monitoring and tracking. With the advance of VLSI designs, we can find consumer sensors for every type of measurements in PSG. Low cost sensors are available for electroencephalogram (EEG), electrooculogram (EOG), electrocardiogram (ECG), electromyogram (EMG), oxygen level, and breathing patterns. It is also very easy to use sound recording functions on smart phones and tablets. With additional data mining and learning software support, it is possible to provide reasonable screening using a set of low end sensors to perform sleep monitoring and tracking. We will further illustrate this step in the following sections. 


\section{Step 2: from wearables to internal body changes}

Step 2 is the transition to bridge the gap between sleep monitoring/ tracking and changes in body biochemistry, and thus leads to new advances in sleep medicine. Sleep is a complicated process and there have been no definitive biomarkers for sleep quality until recently when oxalic acid [generated by processing food] and diacylglycerol 36:3 [fat storage molecule] were shown to be quantitative sleep biomarkers in blood. ${ }^{7}$ These studies suggest that one function of sleep is to clear metabolites in the brain and reinstate an antioxidant balance in peripheral tissues. Loss of sleep causes a harmful oxidative metabolic state. This discovery makes it possible to track the biochemical changes inside the body and correlate these with sleep measurements to discern ways to improve sleep quality for each individual (Figure 2).

Figure 1 displays performance comparisons of PSG, Actiwatch, Basis, and Jawbone. Figure 1 describes the data measurements we have with Actiwatch, Basis, and Jawbone. According to, ${ }^{8,9}$ the three popular devices which collect accurate signals for sleep monitoring/ tracking include Actiwatch, Basis watch, and Jawbone Up. Actiwatch uses a highly accurate MEMS based accelerometer to measure body movements ${ }^{10,11}$ which are then used to determine sleep cycles. Basis Watch measures activity, heart rate (HR), body temperature, and blood flow. It also utilizes software to integrate sensor data and perform analysis to evaluate sleep quality. Jawbone uses a 3D accelerometer to measure acceleration. It also collects heart rate. Both acceleration and heart rate are used to interpret sleep quality via data analysis tools.

Acceleration measurements have limited accuracy. The linear range of acceleration is usually within $\pm 3 \mathrm{~g}$. They are also nonlinear which means that there is a deviation between output voltage and actual acceleration. Other sources of errors are discussed in. ${ }^{12}$ Sensors alone do not provide the infrastructure to store data and perform real time analysis. IoT is required to be in place to connect sensors with data mining and storage capability. Figure 3 discusses how data can be streamed to remote locations using mobile platforms. Figures 4 \& Figure 5 demonstrate screenshots from a sleep framework called "MySleep". ${ }^{13}$ Designed with MySQL database, this sleep framework uploads user data through the website, and facilitates basic data mining capabilities to allow users to manage their sleep time.

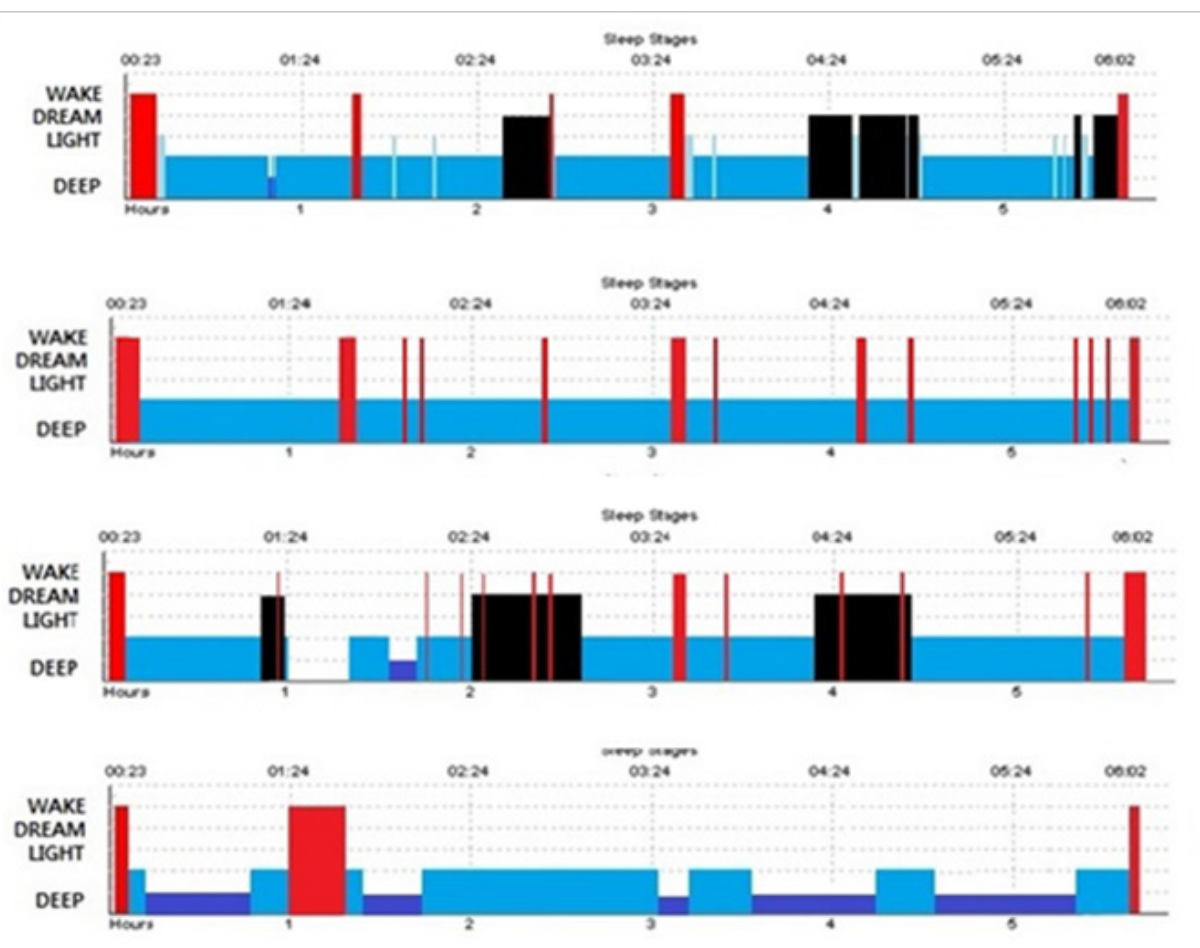

Figure I Sleep tracking result comparison among Polysonogram, Actiwatch, Basis, and Jawbone [6].

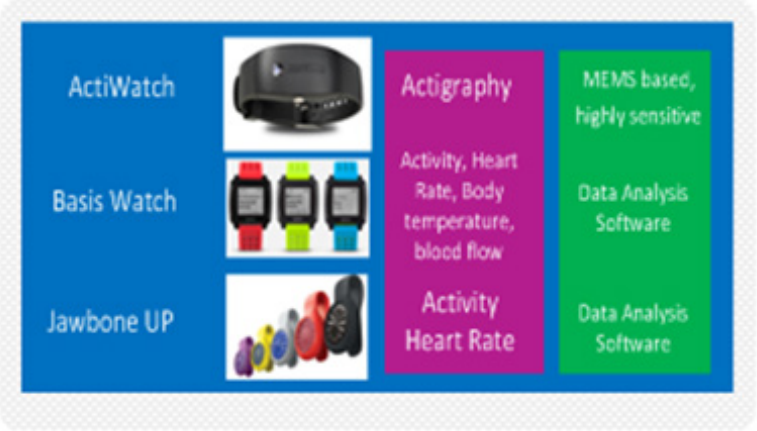

Figure 2 Three sleep tracking/monitoring wristbands.

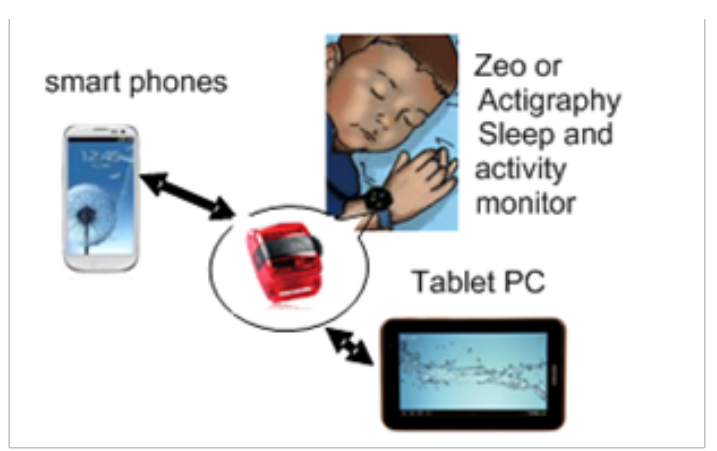

Figure 3 Data streamed to remote locations through loT using a sleep tracking platform. 


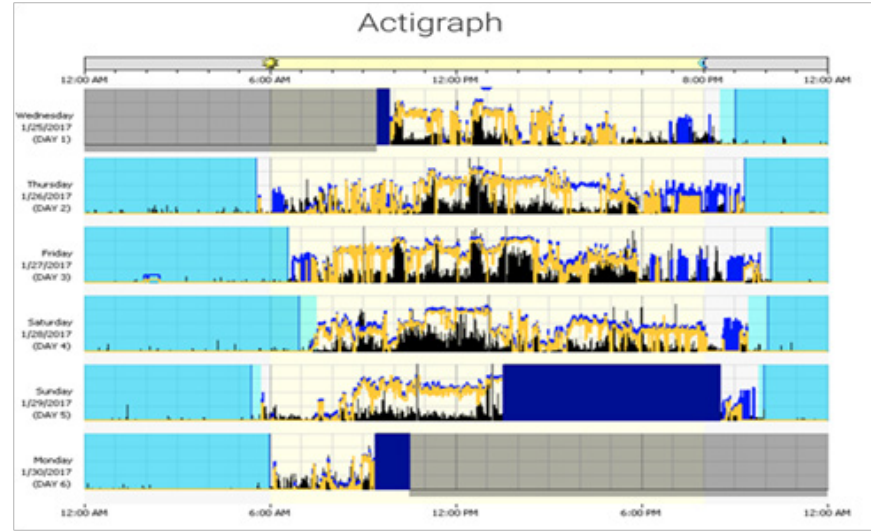

Figure 4 The actigraph for one week from Actiwatch uploaded on "MySleep" framework. This result is from research supported in part by NSF award \#1433185 (collaborators: Michelle Perfect, Janet M. Roveda, and Stuart Quan).

\begin{tabular}{|c|c|c|c|c|}
\hline \multicolumn{5}{|c|}{ Daily Statistics } \\
\hline $\begin{array}{l}\text { Start } \\
\text { Date_Aetigraphy }\end{array}$ & $\begin{array}{l}\text { Bed } \\
\text { Time_Actigraphy }\end{array}$ & $\begin{array}{l}\text { Wake Up } \\
\text { Time_Actigraphy }\end{array}$ & $\begin{array}{l}\text { Time in Bed } \\
\text { (hours:min)_Actigraphy }\end{array}$ & $\begin{array}{l}\text { Total Sleep } \mathrm{TI} \\
\text { (hours:min) } y\end{array}$ \\
\hline Wed $1 / 25 / 2017$ & $8: 31: 30 \mathrm{PM}$ & $534.00 \mathrm{AM}$ & 0902 & $07: 25$ \\
\hline Thu $1 / 26 / 2017$ & $9.20: 30 \mathrm{PM}$ & 6.35.30 AM & $09: 15$ & 08.41 \\
\hline $\mathrm{Fri} 1 / 27 / 2017$ & $1004: 00$ PM & $729: 30$ AM & 0925 & $08: 10$ \\
\hline Sat $1 / 28 / 2017$ & $9: 27: 30 \mathrm{PM}$ & $5: 42.00 \mathrm{AM}$ & $08: 14$ & $06: 49$ \\
\hline Sun $1 / 29 / 2017$ & $9: 46: 30 \mathrm{PM}$ & $5.59 .30 \mathrm{AM}$ & 08.13 & $07: 32$ \\
\hline
\end{tabular}

\section{Actigraphy Summary Statistic}

\begin{tabular}{lllll}
$\begin{array}{l}\text { Earliest Bed } \\
\text { Time_Actigraphy }\end{array}$ & $\begin{array}{l}\text { Latest Bed } \\
\text { Time_Actigraphy }\end{array}$ & $\begin{array}{l}\text { Average Bed } \\
\text { Time_Actigraphy }\end{array}$ & $\begin{array}{l}\text { Earliest Wake } \\
\text { Time_Actigraphy }\end{array}$ & $\begin{array}{l}\text { Latest Wake } \\
\text { Time_Actigraphy }\end{array}$ \\
\hline 083130 PM & 10.0400 PM & 0925.59 PM & $05.34: 00$ AM & $07.29: 30$ AM
\end{tabular}

Figure 5 Statistic summary screenshot for the sleep actigraphy from "MySleep" framework. This result is from research supported in part by NSF award \#|433185 (collaborators: Michelle Perfect, Janet M. Roveda, and Stuart Quan).

\section{Conclusion}

This paper discussed two transitions in sleep quality and reviewed existing wearable sensors that can provide sleep cycle measurements to indicate sleep deficiency. We also presented a new sleep framework that facilitates an IoT structure for sleep monitoring and tracking. This approach is useful for both adults and children. However, further work is needed to enhance the performance and data quality as well as the design of current wearable sensors. It is also important to address data management and security. ${ }^{14}$

\section{Acknowledgements}

None.

\section{Conflict of interest}

The author declares no conflict of interest.

\section{References}

1. Multistate Outbreak of Shiga toxin-producing Escherichia coli O157:H7 Infections Linked to I.M. Healthy brand soynut butter; 2017.

2. Carl P Wonders. NIH consortium takes aim at vascular disease-linked cognitive impairment and dementia; 2017.

3. Overview of sleep disorders. Remedy health Communities.com; 2016.

4. Crupi R, Faetti T, Paradiso R. Preliminary evaluation of wearable wellness system for obstructive sleep apnea detection. Conf Proc IEEE Eng Med Biol Soc. 2015;2015:4142-4145.

5. Hamada M, Iida M. Home monitoring using portable polygraphy for perioperative assessment of pediatric obstructive sleep apnea syndrome. Tokai J Exp Clin Med. 2012;37(3):66-70.

6. Gay PC, Selecky PA. Are sleep studies appropriately done in the home? Respir Care. 2010;55(1):66-75.

7. Weljie A, Meerlo P, Goel N, et al. Oxalic acid and diacylglycerol 36:3 are cross-species markers of sleep debt. Proc Natl Acad Sci. 2015;112(8):2569-2574.

8. The Ultimate Guide To Sleep Tracking; 2017.

9. Christopher Winter. Personal sleep monitors: Do They Work?; 2014.

10. Lambiase MJ, Gabriel KP, Chang YF, et al. Utility of actiwatch sleep monitor to assess waking movement behavior in older women. Med Sci Sports Exerc. 2014;46(12):2301-2307.

11. Kushida CA, Chang A, Gadkary C, et al. Comparison of actigraphic, polysomnographic, and subjective assessment of sleep parameters in sleep-disordered patients. Sleep Med. 2001;2(5):389-396.

12. Accelerometer specifications-quick definitions.

13. MySleep; 2016.

14. Morgenthaler T, Alessi C, Friedman L. Practice parameters for the use of actigraphy in the assessment of sleep and sleep disorders: an update for 2007. Sleep. 2007;30(4):519-529. 